\title{
Influence of stress and health-behaviour on miRNA expression
}

\author{
YORI GIDRON $^{1}$, MARTINA DE ZWAAN $^{2}$, KARL QUINT $^{3,4}$ and MATTHIAS OCKER ${ }^{3,4}$ \\ ${ }^{1}$ Faculty of Medicine and Pharmacy, Free University of Brussels (VUB), Jette, Belgium; \\ Departments of ${ }^{2}$ Psychosomatics and Psychotherapy, and ${ }^{3}$ Medicine 1, University Hospital Erlangen, Erlangen; \\ ${ }^{4}$ Institute for Surgical Research, Philipps University Marburg, Marburg, Germany
}

Received December 1, 2009; Accepted February 4, 2010

DOI: $10.3892 / \mathrm{mmr} 00000279$

\begin{abstract}
Psychological stress is correlated with and may even cause DNA damage, which contributes to the etiology of various diseases. Recent studies point to the role of micro-RNA (miRNA), small molecules that regulate gene expression, in health and disease. This study investigated the relationship between transient stress and two cancer-related miRNAs, and determined whether health-behaviour moderated these relationships. Using a pre-post design, 37 German students completed measures on health-behaviour and perceived stress, the latter after a study break (low stress) and after an exam (high stress). On both occasions, students underwent blood tests to determine the expression of let-7b and miR-21, two miRNAs recently found to be related to cancer. The students reported significantly higher stress after the exam than in the study break period. The levels of let-7b and miR-21 expression significantly declined from low- to high-stress periods. Importantly, baseline health-behaviour interacted with time in relation to miR-21, such that the expression of this marker decreased only in students with inadequate health-behaviour, while it did not change in students with adequate healthbehaviour. This is the first study showing that brief academic stress can alter the expression of two cancer-related miRNA molecules, and that health-behaviour may moderate these effects for miR-21.
\end{abstract}

\section{Introduction}

Psychological stress slows down wound healing and may increase the risk of illness, including infectious and cardiovascular diseases $(1,2)$. The field of psychoneuroimmunology has led scientific efforts to attempt to try and explain such relationships, mainly by focusing on neuroimmune mediators $(2,3)$. However, stress-induced alterations in DNA integrity (4) may also partly explain such relationships at the molecular level, given the known role of DNA damage in disease (5).

Correspondence to: Professor Yori Gidron, Faculty of Medicine and Pharmacy, Vrije Universiteit Brussel, Laarbeeklaan 103, 1090 Jette, Belgium

E-mail: yori.gidron@vub.ac.be

Key words: micro-RNA, psychological stress, health-behaviour
Recent research has discovered novel endogenous regulators of gene expression, namely micro-RNAs (miRNAs). miRNAs are small non-coding RNA molecules that negatively regulate gene expression (6) and play roles in development, health and various illnesses (7). In carcinogenesis, the miRNA let-7b has tumour suppressor properties, while miR-21 seems to enhance oncogenesis $(6,8)$. Psychoneuroimmunological pathways have been proposed to link psychological factors with the onset and progression of cancer (9). However, the effects of psychological stress on these tumour-related miRNAs in healthy people have not been investigated, though they may provide some insight into the role of psychological factors in cancer progression. Furthermore, no study has identified any protective factors that may potentially moderate any effects of stress on miRNAs. A healthy lifestyle (e.g., physical exercise and diet) was found to reduce the effects of various risk factors (e.g., hypertension) on disease risk (heart disease) (10). A healthy lifestyle similarly could protect intracellular factors from adverse stress effects. The present study investigated the relationship between transient (academic exam) stress and cancer-related miRNA expression (let-7b and miR-21), and determined whether health-behaviour moderates the effects of stress on the expression of these miRNAs.

\section{Materials and methods}

Design. A within-subject pre-post design was used, where students served as their own controls. Students were assessed on perceived stress and micro-RNAs after the semester break (low stress) and soon after exiting an exam (high stress). They also provided, at baseline, data on various background information, including health-behaviour.

Participants. Thirty-seven students (28 female, 9 male) studying medicine at Erlangen University, Germany, with a mean age of 22.7 (SD 2.2) years were included in the study. The present study was approved by the local ethics committee.

Measures. At baseline (and after the exam), students were assessed for health-behaviour (e.g., physical exercise, diet and stress control) using a 13-item validated questionnaire that yields a single score (11). Health-behaviour scores at baseline and during the exam period were highly correlated $(r=0.72$, $\mathrm{p}<0.001$ ), supporting the stability of this measure. Students underwent assessment of perceived stress at baseline and 
following the exam, using the perceived-stress questionnaire (PSQ; e.g., irritability, tension and overload) (12). The internal reliability of the PSQ at baseline and after the exam was very high (Cronbach's $\alpha, 0.92$ and 0.91, respectively), supporting the reliability of this measure.

To determine the expression of let-7b and miR-21, the students provided blood samples. These were processed by the miRNeasy kit and converted to cDNA using the miScript Reverse Transcription kit (both from Qiagen, Hilden, Germany). Samples were run on a LightCycler system with the miScript SYBR Green PCR kit and miScript Primer assays (both from Qiagen). For each sample, the results were adjusted to the content of RNU6B, a commonly expressed short endogenous miRNA $45 \mathrm{nt}$ in length without any known corresponding target gene, as a general index of synthesis of miRNAs.

Statistical analysis. Distributions of miRNAs were normalized using log-transformations. We used paired t-tests to test the effects of time period and analyses of variance (ANOVA) to test whether baseline health-behaviour interacted with time (calm/stress periods) in relation to miRNAs. SPSS 14 software was used for statistical analyses.

\section{Results}

Background variables (age, gender and health-behaviour) were not significantly related to miRNA variables at any time point (data not shown), and the inclusion of the first two as covariates in the analyses did not alter the results. Hence, the results reported here do not consider these variables as covariates. The students reported significantly lower stress levels during the low-stress period (mean 81.60, SD 11.63) than during the high-stress period (mean 85.23, SD 11.42), $\mathrm{t}(34)=2.24, \mathrm{p}=0.032$ (2-tailed). As shown in Fig. 1, the levels of let-7b significantly declined between the low-stress period (mean 0.52, SD 0.79) and the high-stress period (mean 0.23, SD 1.09), $\mathrm{t}(36)=2.15$, $\mathrm{p}=0.04$ (2-tailed). Similarly, the levels of miR-21 significantly declined between the low-stress period (mean 0.96, SD 0.92) and the high-stress period (mean -0.31, SD 1.35), $\mathrm{t}(36)=2.69$, $\mathrm{p}=0.01$ (2-tailed) (Fig. 1).

We then examined whether the baseline health-behaviour scores moderated the effects of time on miRNAs. ANOVA revealed that baseline health-behaviour (low/high at the median) significantly interacted with time (low/high stress periods) in relation to miR-21 only $[\mathrm{F}(1,34)=8.49, \mathrm{p}<0.01]$ (Fig. 2). In students with adequate health-behaviour (above the median), no change in miR-21 was noted over time ( $\mathrm{p}=0.84)$. However, in students with initially poorer health-behaviour, miR-21 declined between the low- and high-stress periods ( $\mathrm{p}=0.007$ ). A similar pattern was also noted when using continuous scores of health-behaviour. No such health-behaviour $\mathrm{x}$ time interaction was noted for let- $7 \mathrm{~b}[\mathrm{~F}(1,34)=1.19, \mathrm{p}>0.05]$.

\section{Discussion}

To the best of our knowledge, this study is the first to investigate in humans the relationship between psychological stress and expression of miRNAs that regulate cancer-related genes. The results demonstrated that exam stress is related to a

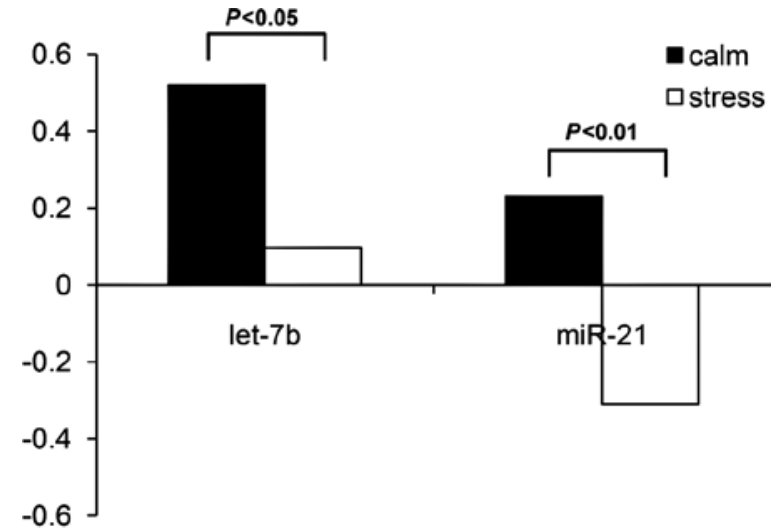

Figure 1. Effects of stress on the expression of miRNAs let-7b and miR-21. The mean expression levels of miRNAs normalized to the levels of housekeeping miRNA RNU6B under non-stressed (calm) or stressed conditions are shown.

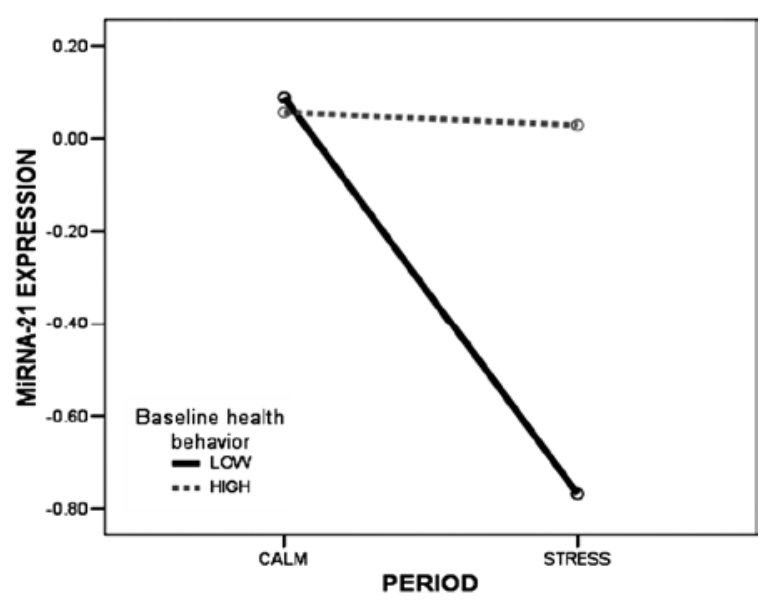

Figure 2. Effects of baseline health-behaviour and time period on the expression of miR-21. Stress reduced miR-21 levels only in individuals with a low health-behaviour score (bold line), but not in those with a high baseline health-behaviour score (dashed line). The mean miR-21 expression normalized to RNU6B levels for each sample is shown.

reduction in the expression of let- $7 \mathrm{~b}$ and $\mathrm{miR}-21$, and that the reduction noted in miR-21 is moderated by initial adequate health-behaviour. These results extend those on stress and general DNA damage (4) to cancer-related miRNAs in healthy people. We are uncertain why both markers declined with stress. It is important to note that the sample in the present study included young healthy people, not cancer patients. In healthy people, it is possible that the expression of miR-21 is required to prevent cell death or mediate repair processes in cells possibly damaged by stress. The observed moderating role of health-behaviour on stress-induced reductions in miR-21 expression supports our interpretation that miR-21 expression may be desirable in healthy people. A similar moderation of the effects of risk factors on adverse health outcomes was observed in a study showing that adequate health-behaviour reduced the cardiac risk associated with hypertension (10). In addition to the oncogeneic properties of miR-21, a role during embryonic development has recently been described (13). As the expression of miR-21 may be transcriptionally regulated via the ubiquitous transcription factor 
AP-1, the sustained miR-21 expression in the individuals with good health-behaviour despite stress supports the notion that miR-21 can also protect cells from stress-induced apoptosis (14). Regarding healthy cancer-free individuals, the oncogeneic (anti-apoptotic) properties of miR-21 may therefore protect tissues from stress-induced damage responses (e.g., after the formation of reactive oxygen species and DNA double-strand breaks) (4), enabling cells to survive following DNA-damage repair.

The main limitation of the present study was that it did not use an experimental design or a control group of students not exposed to stress who could have been measured twice for miRNA expression levels. Future experimental studies should test whether the results obtained in the present study confirm a causal relation between psychological stress and changes in miRNA.

In summary, we found that even a transient and relatively mundane stressor (exam stress) may affect cancer-related modulators of gene expression, and that adequate healthbehaviour may partly moderate this. Further miRNA array analyses are needed to determine miRNA profiles influenced by psychological stress, and the possible implications of such changes to health. The present study did not use an experimental design; future experimental studies should verify whether the results obtained confirm a causal relationship between psychological stress and changes in miRNA. Future studies also need to ascertain whether psychological stress alters the cancer-related genetic targets of these and other miRNAs (e.g., p53). Given that miRNAs have also been related to psychiatric illnesses such as schizophrenia (15), the implications of these findings on stress-induced mental diseases deserve further attention as well. Finally, future studies should focus on whether psychological stress, health-behaviour and the expression of miRNAs interact in predicting physical and psychiatric disease onset and progression. The preliminary findings observed here suggest that possible stress-induced changes in miRNAs need to be further investigated as possible mediators between psychological stress and the onset and progression of illnesses.

\section{References}

1. Cohen S: Keynote presentation at the Eight International Congress of Behavioural Medicine: the Pittsburgh common cold studies: psychosocial predictors of susceptibility to respiratory infectious illness. Int J Behav Med 12: 123-131, 2005.

2. Godbout JP and Glaser R: Stress-induced immune dysregulation: implications for wound healing, infectious disease and cancer. J Neuroimmune Pharmacol 1: 421-427, 2006.

3. Greeson JM, Hurwitz BE, Llabre MM, Schneiderman N, Penedo FJ and Klimas NG: Psychological distress, killer lymphocytes and disease severity in HIV/AIDS. Brain Behav Immun 22: 901-911, 2008.

4. Gidron Y, Russ K, Tissarchondou $\mathrm{H}$ and Warner J: The relation between psychological factors and DNA-damage: a critical review. Biol Psychol 72: 291-304, 2006.

5. Wu LL, Chiou CC, Chang PY and Wu JT: Urinary 8-OHdG: a marker of oxidative stress to DNA and a risk factor for cancer, atherosclerosis and diabetics. Clin Chim Acta 339: 1-9, 2004

6. Jerome T, Laurie P, Louis B and Pierre C: Enjoy the silence: the story of let-7 microRNA and cancer. Curr Genomics 8: 229-233, 2007.

7. Bicker S and Schratt G: microRNAs: tiny regulators of synapse function in development and disease. J Cell Mol Med 12: 1466-1476, 2008.

8. Nam EJ, Yoon H, Kim SW, et al: MicroRNA expression profiles in serous ovarian carcinoma. Clin Cancer Res 14: 2690-2695, 2008.

9. Gidron $\mathrm{Y}$ and Ronson A: Psychosocial factors, biological mediators and cancer prognosis: a new look at an old story. Curr Opin Oncol 20: 386-392, 2008.

10. Pitsavos C, Panagiotakos DB, Chrysohoou C, et al: The effect of the combination of Mediterranean diet and leisure time physical activity on the risk of developing acute coronary syndromes, in hypertensive subjects. J Hum Hypertens 16: 517-524, 2002.

11. Bertera EM: Development and validation of a short healthbehaviours index for use with low socioeconomic status (SES) older adults. J Clin Geropsych 8: 101-115, 2002.

12. Levenstein S, Prantera C, Varvo V, et al: Development of the Perceived Stress Questionnaire: a new tool for psychosomatic research. J Psychosom Res 37: 19-32, 1993.

13. Hu SJ, Ren G, Liu JL, et al: MicroRNA expression and regulation in mouse uterus during embryo implantation. J Biol Chem 283: 23473-23484, 2008.

14. Chan JA, Krichevsky AM and Kosik KS: MicroRNA-21 is an antiapoptotic factor in human glioblastoma cells. Cancer Res 65: 6029-6033, 2005.

15. Beveridge NJ, Tooney PA, Carroll AP, et al: Dysregulation of miRNA $181 \mathrm{~b}$ in the temporal cortex in schizophrenia. Hum Mol Genet 17: 1156-1168, 2008. 